\title{
Microscopic calculations of the characteristics of radiative nuclear reactions for double-magic nuclei
}

\author{
Oleg Achakovskiy ${ }^{1, a}$, Sergei Kamerdzhiev², Victor Tselyaev ${ }^{3}$, and Mikhail Shitov ${ }^{4}$ \\ ${ }^{1}$ Institute for Physics and Power Engineering, Obninsk, Russia \\ ${ }^{2}$ National Research Centre "Kurchatov Institute", Moscow, Russia \\ ${ }^{3}$ Physical Faculty, St. Petersburg State University, St. Petersburg, Russia \\ ${ }^{4}$ National Research Nuclear University MEPhl, Moscow, Russia
}

\begin{abstract}
The neutron capture cross sections and average radiative widths $\Gamma_{\gamma}$ of neutron resonances for two double-magic nuclei ${ }^{132} \mathrm{Sn}$ and ${ }^{208} \mathrm{~Pb}$ have been calculated using the microscopic photon strength functions (PSF), which were obtained within the microscopic self-consistent version of the extended theory of finite Fermi systems in the time blocking approximation. For the first time, the microscopic PSFs have been obtained within the fully self-consistent approach with exact accounting for the single particle continuum (for ${ }^{208} \mathrm{~Pb}$ ). The approach includes phonon coupling effects in addition to the standard RPA approach. The known Skyrme force has been used. The calculations of nuclear reaction characteristics have been performed with the EMPIRE 3.1 nuclear reaction code. Here, three nuclear level density (NLD) models have been used: the so-called phenomenological GSM, the EMPIRE specific (or Enhanced GSM) and the microscopical combinatorial HFB NLD models. For both considered characteristics we found a significant disagreement between the results obtained with the GSM and HFB NLD models. For ${ }^{208} \mathrm{~Pb}$, a reasonable agreement has been found with systematics for the $\Gamma_{\gamma}$ values with HFB NLD and with the experimental data for the HFB NLD average resonance spacing $D_{0}$, while for these two quantities the differences between the values obtained with GSM and HFB NLD are of several orders of magnitude. The discrepancies between the results with the phenomenological EGLO PSF and microscopic RPA or TBA are much less for the same NLD model.
\end{abstract}

\section{Introduction}

In order to calculate characteristics of nuclear reactions with gamma-rays, the information is necessary, at least, about the photon strength function (PSF) and nuclear level density (NLD) models. Traditionally, these quantities have been parametrized phenomenologically with the parameters fitted for stable nuclei. For example, the PSF has been parametrized on the basis of smooth Lorentzian type functions but, as it was noted in [1,2], these phenomenological Lorentzian-type expressions for PSF are not able to predict the observed structures (under that condition that the Brink-Axel hypothesis is true). Also, the shortcomings of analytical NLD formulae in matching experimental data are overcome, as a rule, by empirical parameter adjustments. For these reasons, the application of phenomenological models for PSF and NLD to nuclei far from the stability valley is questionable $[1,2]$.

However, there are also questions of this type for double-magic nuclei. The problem is that the phenomenological approaches "smooth" the individual characteristics of these nuclei or consider them on the average. Individual peculiarities are especially expressive just for double-magic nuclei, even for stable, not to mention un-

\footnotetext{
a e-mail: oachakovskiy@ippe.ru
}

stable those, whose properties can be unknown. For example, to include the vibrational NLD enhancement to the well-known so-called generalized superfluid model (GSM) [1, 2], the experimental values for the energies of the first $2^{+}$and the formula $50 A^{-2 / 3} \mathrm{MeV}$ for the first $3^{-}$levels are used. The formula is not suited for doublemagic nuclei, and both of these prescriptions should not be suited for unstable nuclei. The microscopic approach in the nuclear theory accounts for specificity of each nucleus through its single-particle and collective (phonon) spectra. Therefore, it allows "some irregular changes" obtained in the global phenomenological models for nuclear reactions data [3] to be seen and checked. Thus, for double-magic nuclei it is necessary to use the microscopic approaches for both PSF and NLD.

In this work, we have applied for PSF the selfconsistent version of microscopic extended theory of finite Fermi systems (ETFFS) [4] in the time blocking approximation (TBA) [5]. For NLD, we used the phenomenological GSM [1], the EMPIRE specific NLD model [6] and the microscopic HFB plus combinatorial NLD model [7]. The calculations of neutron capture cross sections and average radiative widths of neutron resonances for two doublemagic nuclei - the stable ${ }^{208} \mathrm{~Pb}$ and unstable ${ }^{132} \mathrm{Sn}$ - have been made using the EMPIRE 3.1 nuclear reaction code. 


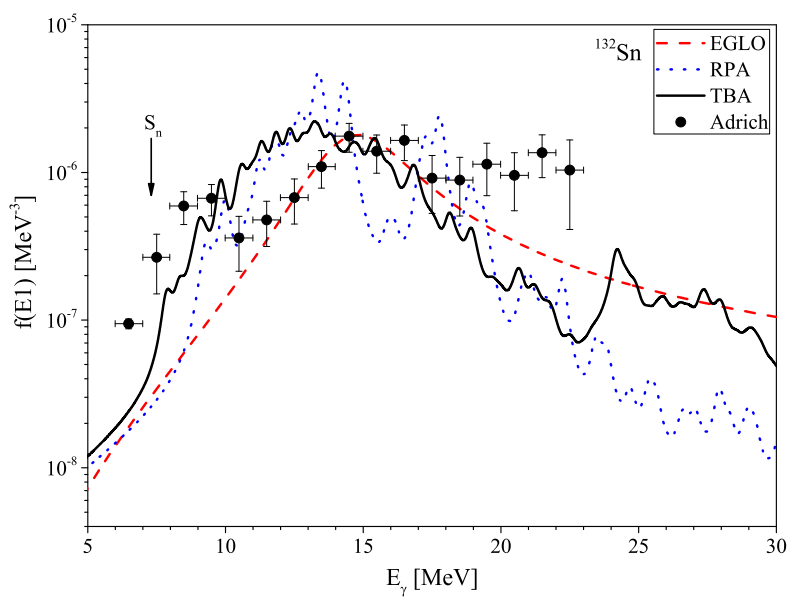

Figure 1. (Color online) The PSF for ${ }^{132} \mathrm{Sn}$. Dotted lines correspond to the self-consistent RPA, solid lines to the TBA (including PC), and dashed lines to the EGLO model [1]. Experimental data [8] were recalculated by us for PSF.

The comparison with the phenomenological PSF EGLO model has been also performed. In all the PSF calculations the smoothing parameter $200 \mathrm{keV}$ has been used. See the details of the calculations in [9] and [10].

Quite recently, the fully self-consistent calculations of giant resonances [11] have been realized for double-magic nuclei within both RPA and TBA. As a new feature in these calculations, the single-particle continuum was included, thus avoiding the artificial discretization usually implied in RPA and TBA. In our previous calculations for semimagic nuclei, see, for example, $[9,10,12]$, a discretization procedure for the single-particle continuum was used (which gave the same results within CRPA for doublemagic nuclei [9]). As the self-consistent TBA calculations of photoabsorption or PSF are rather time-consuming, we have used the corresponding results for ${ }^{132} \mathrm{Sn}$ from Ref. [9] and for ${ }^{208} \mathrm{~Pb}$ from [11] obtained with the Skyrme forces SLy4 to calculate the above mentioned nuclear reaction characteristics.

\section{Photon strength functions}

In Fig. 1 and Fig. 2 we show the PSFs for ${ }^{132} \mathrm{Sn}$ and ${ }^{208} \mathrm{~Pb}$ calculated within our microscopic (ETFFS(TBA)), or simply TBA, and RPA methods with Skyrme forces SLy4. These PSFs have been recalculated from the theoretical photoabsorption cross sections taken from [9] $\left({ }^{132} \mathrm{Sn}\right)$ and [11] $\left({ }^{208} \mathrm{~Pb}\right)$. The phenomenological EGLO PSFs are also shown. In Fig. 1, the $E 1 \mathrm{PSF}$ for ${ }^{132} \mathrm{Sn}$ is compared with experimental data from Ref. [8]. In Fig. 2, the $E 1$ PSF for ${ }^{208} \mathrm{~Pb}$ is compared with the experimental data obtained within the Oslo method [13]. As it can be seen in contrast to the phenomenological model EGLO and microscopic CRPA, the CTBA approach, i.e. RPA + phonon coupling, can describe some observed structures of PDR in ${ }^{132} \mathrm{Sn}$ and partly ${ }^{208} \mathrm{~Pb}$ only due to phonon coupling. For ${ }^{132} \mathrm{Sn}$, we see the well-known structure at about 10 $\mathrm{MeV}$ (our approach gives a lower energy), usually called

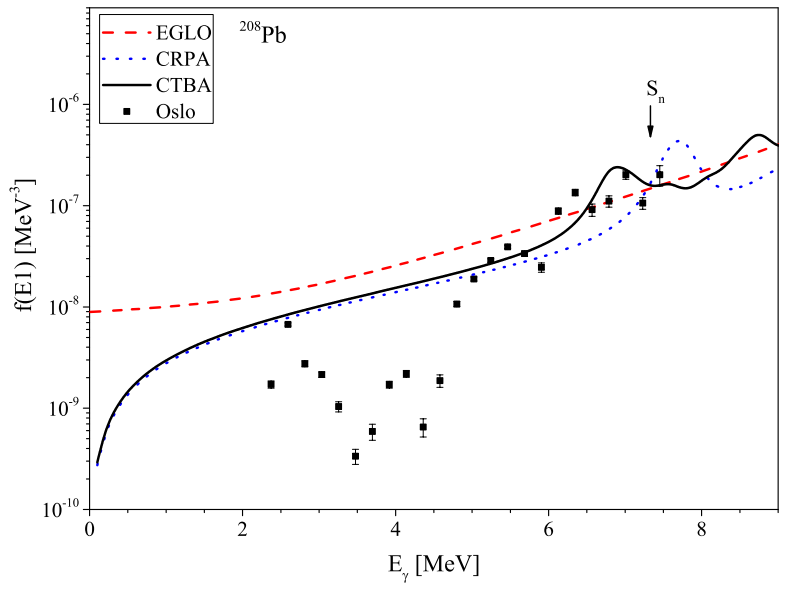

Figure 2. (Color online) E1 PSF for ${ }^{208} \mathrm{~Pb}$. Dotted lines correspond to the self-consistent CRPA, solid lines to the CTBA (including PC), and dashed lines to the EGLO model [1]. Experimental data are taken from Ref. [13].

as pygmy-dipole resonance for the photoabsorption cross section, see the discussions in $[9,14,15]$.

Let us discuss the results shown in Fig. 2 for ${ }^{208} \mathrm{~Pb}$. We see that the CTBA approach describes the experiment on the whole at $E>5 \mathrm{MeV}$ and does it better than CRPA (note that the smoothing parameter $200 \mathrm{keV}$ has been used in the calculations). However, we have a large disagreement with experimental data at $E<5 \mathrm{MeV}$. As one can see from [16], where the transitions between ground end excited states have been measured, the beginning of the $1^{-}$ excitation spectrum in [16] is $4.84 \mathrm{MeV}$, i.e. there is no $1^{-}$ transitions between ground and excited states below 4.84 $\mathrm{MeV}$. This result is understandable: in the double-magic ${ }^{208} \mathrm{~Pb}$ there is no single-particle or two-phonon $E 1$ transitions at about $E<5 \mathrm{MeV}$.

In order to obtain some additional information we have compared with each other two sets of experimental data for ${ }^{208} \mathrm{~Pb}$ (see Fig. 3) : 1) the PSF data from [13] where the transitions between ground and excited states as well as between excited states could be measured and 2) the data [16] for the $B(E 1)$ values for the transitions between only ground and excited states. It is necessary to compare both sets of data with approximately the same smoothing. So, taking into account that the experimental resolution in the experimental data [13] is about $200 \mathrm{keV}$, we smoothed the data [16] with three smoothing parameters $100 \mathrm{keV}$, $200 \mathrm{keV}$, and $400 \mathrm{keV}$. As it can be expected, we obtained a rough agreement between both sets of experimental data at $E>4.84 \mathrm{MeV}$. Thus, one can think that the excitations observed in [13] at $E<4.84 \mathrm{MeV}$ are only caused by transitions between excited states. However, it is necessary to underline that the mechanisms of the reactions used in [13] and [16] are very different and the use of energy-independent smoothing parameter may be too approximate. 


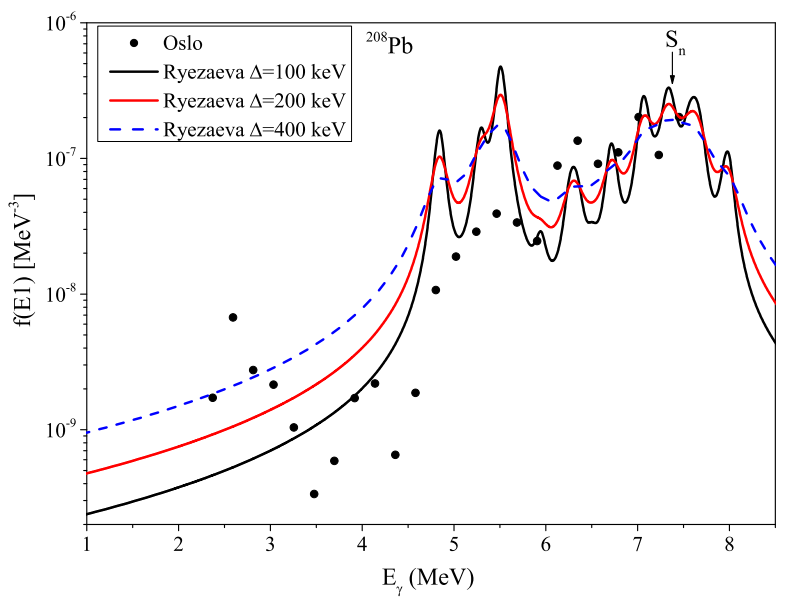

Figure 3. (Color online) Comparison of the experimetal data: the $\left({ }^{3} \mathrm{He},{ }^{3} \mathrm{He} \gamma\right)$ reactions method [13] and nuclear resonance fluorescence technique [16]. The lowest $1^{-}$-level of the data [16] is 4.84 MeV. It was smoothed by us with three smoothing parameters $\Delta$. See text for details.

\section{Neutron capture cross sections}

In Fig. 4 and Fig. 5, the neutron radiative capture cross sections are shown for the compound ${ }^{132} \mathrm{Sn}$ and ${ }^{208} \mathrm{~Pb}$. Our approach for PSF is non-statistical, so there is no sense to compare its results with the available ${ }^{207} \mathrm{~Pb}(n, \gamma){ }^{208} \mathrm{~Pb}$ cross sections $[17,18]$ because these data (two points) are in the neutron resonance energy region. We see a very large difference between the results obtained with traditional GSM and other NLD models (EMPIRE specific and HFB+combinatorial), namely, the difference for $(n, \gamma)$ cross sections is about one order of magnitude practically at all the neutron energy up to $2 \mathrm{MeV}$ and $10 \mathrm{MeV}$ for the compound ${ }^{132} \mathrm{Sn}$ and ${ }^{208} \mathrm{~Pb}$, respectively. There is no noticeable difference between the results with phenomenological EMPIRE specific and microscopic HFB+combinatorial NLD models. One of the possible reasons is that in both cases the known experimental energies of the first $2^{+}$levels have been used which is, generally speaking, important for the phonon enhancement effect in the NLDs (it is also useful to note that the energy of the first $2^{+}$level in ${ }^{132} \mathrm{Sn}$ calculated self-consistently in our TBA is $4.34 \mathrm{MeV}$ while the experimental energy is 4.041 $\mathrm{MeV})$. A detailed discussion about these results will be presented somewhere else, so we showed so many curves in Fig. 4 and Fig. 5 in order to obtain general information.

\section{Average radiative widths}

Unfortunately, the experimental data are very scarce for double-magic nuclei ${ }^{132} \mathrm{Sn}$ and ${ }^{208} \mathrm{~Pb}$. However, for ${ }^{208} \mathrm{~Pb}$ with EMPIRE 3.1 we found, see Table 1, for the average radiative widths $\Gamma_{\gamma}$ values, a reasonable agreement with the systematics [19] only for EMPIRE specific and $\mathrm{HFB}+$ combinatorial NLD models. For the average resonance s-wave level spacings $D_{0}$, the following was found: $D_{0}(\mathrm{GSM})=0.00441 \mathrm{keV}, D_{0}($ EMPIRE specific $)=$

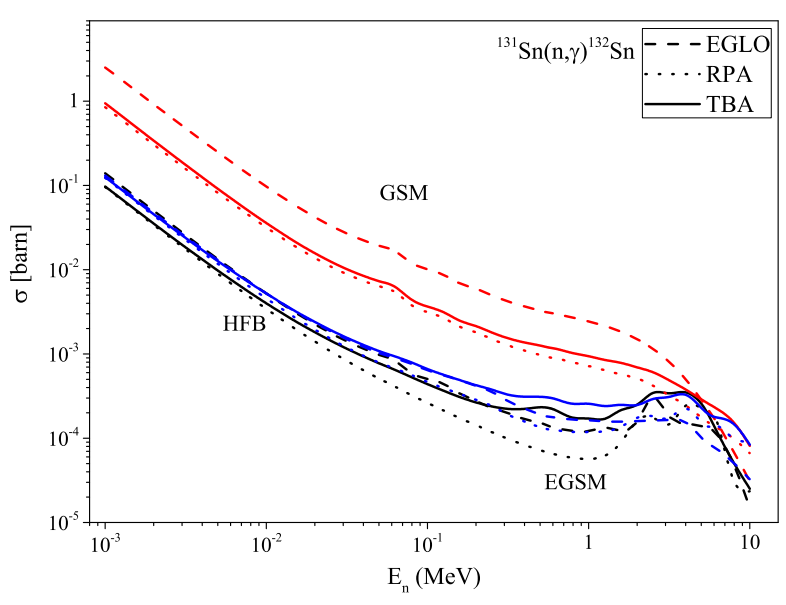

Figure 4. (Color online) ${ }^{131} \mathrm{Sn}(n, \gamma){ }^{132} \mathrm{Sn}$ cross section calculated with the EGLO (dash), RPA (dot) and TBA (solid) PSFs. The red curves were calculated using EMPIRE 3.1 with the GSM NLD model, black ones: the EMPIRE specific NLD and blue ones: the HFB+combinatorial NLD.

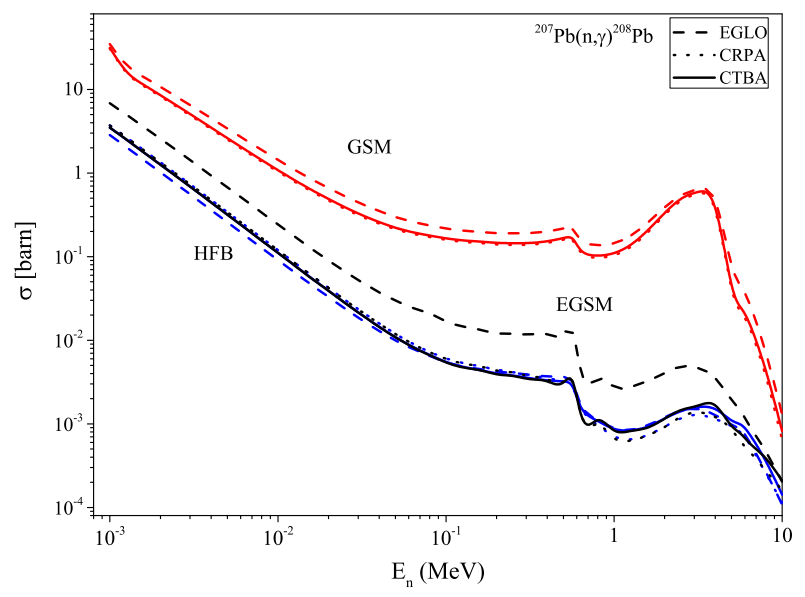

Figure 5. (Color online) The same as for Fig. 4, but for the ${ }^{207} \mathrm{~Pb}(n, \gamma){ }^{208} \mathrm{~Pb}$ cross section calculated with the EGLO (dash), CRPA (dot), and CTBA (solid) PSFs.

$32.0 \mathrm{keV}, D_{0}(\mathrm{HFB})=37.6 \mathrm{keV}$, while $D_{0}(\exp )=$ $30(8) \mathrm{keV}$. The EMPIRE produces unreasonably small value for $D_{0}(\mathrm{GSM})$.

In the last column of Table 1 , the contribution of $M 1$ resonance [1] to $\Gamma_{\gamma}$ calculated with EMPIRE 3.1 is given, which is based on the standard Lorentz approximation with the width $\Gamma=4 \mathrm{MeV}$. It turned out rather small. As discussed in [20], this $\Gamma$ value is very questionable, especially for ${ }^{208} \mathrm{~Pb}$.

\section{Conclusion}

Here, the self-consistent microscopic approach for the PSFs calculations has been used for the double-magic nuclei ${ }^{132} \mathrm{Sn}$ and ${ }^{208} \mathrm{~Pb}$. To calculate neutron radiative cross sections and average radiative widths, we have used the EMPIRE 3.1 code. A noticeable specificity of the con- 
Table 1. Average radiative widths $\Gamma_{\gamma}(\mathrm{meV})$ for s-wave neutrons. Systematics is taken from Ref. [19].

\begin{tabular}{lllllll}
\hline Nuclei & NLD model & EGLO & RPA & TBA & System. & $\begin{array}{l}M 1 \\
\text { contrib. }\end{array}$ \\
\hline \multirow{2}{*}{${ }^{132} \mathrm{Sn}$} & GSM & 398 & 133 & 148 & 40.9 \\
& EMPIRE specific & 7340 & 4675 & 5186 & 515.3 \\
& comb. HFB & 4444 & 4279 & 4259 & 340.7 \\
\hline \multirow{2}{*}{${ }^{208} \mathrm{~Pb}$} & GSM & 10.56 & 4.44 & 4.61 & 5070 & 0.79 \\
& EMPIRE specific & 6292 & 2562 & 2109 & 3770 & 6.56 \\
& comb. HFB & 2734 & 2973 & 2448 & & 5.25 \\
\hline
\end{tabular}

sidered double-magic nuclei has been found. The contribution of the phonon coupling is not so noticeable, on the whole, as compared with the semi-magic nuclei $[9,12]$. For the considered characteristics, a very significant disagreement between the results obtained with the phenomenological GSM and microscopic HFB NLD models has been found. The discrepancies between the results with the phenomenological EGLO PSF and microscopic RPA (or CRPA) or TBA (or CTBA) are much less for the same NLD model.

The results obtained confirm the necessity of using consistent microscopic approaches for calculations of radiative nuclear characteristics in double-magic nuclei. Also, due to comparison of the two sets of experimental data [13] and [16], it was possible to conclude that the nature of the PSF values observed in [16] at $E<4.84 \mathrm{MeV}$ for ${ }^{208} \mathrm{~Pb}$ should be only caused by the transitions between excited states.

\section{Acknowledgements}

The authors acknowledge useful discussions with Drs. A. Voinov and V.G. Pronyaev. V.T. acknowledges the research grant 11.38.648.2013 from Saint Petersburg State University.

\section{References}

[1] T. Belgya, O. Bersillon, R. Capote, et al., Handbook for Calculations of Nuclear Reaction Data, RIPL-2, IAEA-TECDOC-1506 (IAEA, Vienna, 2006) [http://www-nds.iaea.org/RIPL-2/].

[2] R. Capote, M. Herman, P. Oblozinsky, et al., Nucl. Data Sheets 110, 3107 (2009); see also https://wwwnds.iaea.org/RIPL-3.

[3] T.S. Belanova, A.V. Ignatyuk, A.B. Pashchenko, V.I. Plyaskin, Handbook Radiative neutron capture (Energoatomizdat, Moscow, 1986)

[4] S. Kamerdzhiev, J. Speth, G. Tertychny, Phys. Rep. 393, 1 (2004)
[5] V. Tselyaev, Phys. Rev. C 75, 024306 (2007)

[6] M. Herman, R. Capote, B.V. Carlson et al., Nucl. Data Sheets, 108, 2655 (2007); see also http://www.nndc.bnl.gov/empire/index.html.

[7] S. Goriely, S. Hilaire, A.J. Koning, Phys. Rev. C 78, 064307 (2008)

[8] P. Adrich, A. Klimkiewicz, M. Fallot, et al., Phys. Rev. Lett. 95, 132501 (2005)

[9] A. Avdeenkov, S. Goriely, S. Kamerdzhiev, S. Krewald, Phys. Rev. C 83, 064316 (2011)

[10] O.I. Achakovskiy, A.V. Avdeenkov, S.P. Kamerdzhiev, Proceedings of the Inter. Conf. "Nuclear Structure and Related Topics” (NSRT15), EPJ Web of Conf., to be published.

[11] N. Lyutorovich, V. Tselyaev, J. Speth, et al., Phys. Lett. B 749, 292 (2015)

[12] O. Achakovskiy, A. Avdeenkov, S. Goriely, et al., Phys. Rev. C 91, 034620 (2015)

[13] N.U.H. Syed, M. Guttormsen, F. Ingebretsen, et al., Phys. Rev. C 79, 024316 (2009)

[14] D. Savran, T. Aumann, A. Zilges, Prog. Part. Nucl. Phys. 70, 210 (2013)

[15] N. Paar, D. Vretenar, E. Khan, G. Colo, Rep. Prog. Phys. 70, 691 (2007)

[16] N. Ryezayeva, T. Hartmann, Y. Kalmykov, et al., Phys. Rev. Lett. 89, 272502 (2002)

[17] R.C. Greenwood, C.W. Reich, Phys. Rev. C 4, 2249 (1971)

[18] O.A. Wasson, R.E. Chrien, R.C. Greenwood, Rept. USNDC-7 P36.

[19] S.F. Mughabghab, Atlas of neutron Resonances, Resonance Parameters and Thermal Cross Sections $Z=1$ 100 (Elsevier, Amsterdam, 2006)

[20] S.P. Kamerdzhiev, S.F. Kovalev, Phys. At. Nucl. 69, 418 (2006) 